\title{
A General Equation Based on Entropy Generation Minimization to Optimize Plate Fin Heat Sink
}

\author{
Hossain Nemati \\ Department of Mechanics, Marvdasht Branch, Islamic Azad University, Marvdasht, Iran \\ E-mail: H.Nemati@miau.ac.ir
}

\begin{abstract}
In the present study, a new method based on entropy generation minimization was proposed to optimize plate fin heat sink. It is clear that the design of a heat sink is not straightforward and needs some secondary equations. Therefore, in the present study, dimensionless forms of equations were presented first and the effect of each dimensionless group on the entropy generation rate was studied deeply. Finally, a semi-analytical equation was proposed to relate dimensionless thickness to dimensionless fin height and Bi number as well. Interestingly, since no restricting assumption was made during derivation, this equation can be used for both natural and forced convection. Moreover, this equation is based on dimensionless parameters, so it is not limited to some specific geometries or working conditions. At the end, six examples showed benefits and ease of use of this equation. A fan curve was also used in optimizing a heat sink which is more realistic than using a specified free stream velocity.
\end{abstract}

Keywords: Entropy generation minimization, natural convection, forced convection, heat sink.

ENGINEERING JOURNAL Volume 22 Issue 1

Received 10 July 2017

Accepted 11 September 2017

Published 31 January 2018

Online at http:/ /www.engj.org/

DOI:10.4186/ej.2018.22.1.159 


\section{Introduction}

\subsection{Introduction}

Design or selection of a reliable heat sink is not always straightforward and usually, needs enough experience. Many parameters play a crucial role in designing a proper heat sink such as fin height, fin thickness, fin spacing etc. On the other hand, minimizing electronic devices depends also on minimizing the cooling systems, which means lighter and smaller heat sinks are avoidable. Many thermal analysis tools such as empirical correlations [1-6] or numerical methods [7-9] may be utilized to analyze the performance of a heat sink for a given design condition. However, it always needs some constraints or methods to help a designer to select heat sink dimensions. Because a designer is always encountered to this question that what fin height, fin thickness or fin spacing shall be selected finally. Several studies may be founded, concerning minimizing the heat sink resistance. Among different methods for optimization of a heat sink, entropy generation minimization (EGM) is more reliable. It is commonly used to compare thermal flow systems [10-14]. Furukawa and Yang [15] performed CFD to predict the optimum design of a plate fin heat sink in natural convection and showed the closeness of results with EGM prediction. Shish and Liu [16] used EGM to propose a method to optimize a plate fin heat sink for electronic cooling under forced convection flow. Culham and Muzychka [17] later developed that method to optimize a plate fin heat sink under laminar forced convection flow. Although the proposed method is valuable, their work was not ended to any explicit correlation. In the same way, Khan et al. [18-19] used EGM method for optimization of a pin fin heat sink. EMG was also used by other researchers [20].

\subsection{Objectives of Present Study}

All previous works are common in two items. Firstly, fin efficiency is a pre-assumed parameter, while it depends on geometry as well as heat transfer coefficient. Secondly, all works are based on dimensional variables and because of that, all works are ended only to an optimizing procedure and not to any correlation. In the present work, the dimensionless equations are presented first including fin efficiency and then, by minimizing the entropy generation, a new equation is proposed to relate effective dimensionless variables. Since no specific assumption is made, the proposed equation can be used for both natural and forced convection. In the following, required equalities are presented in both original and dimensionless forms and the effect of different variables on entropy generation are studied. Considering the influence of different variables, a new equation is proposed and tested to relate effective dimensionless variables in optimized working condition. Finally, the proposed equation is used in optimizing some heat sinks in different working condition under both free and forced convection.

\section{Model Development}

A typical plate fin heat sink is shown in Fig. 1. According to this figure, the fin thickness is $t$, fin spacing is $b$ and the base of the heat sink is $\mathrm{W} \times \mathrm{L}$. The heat sink base thickness is tb which is not shown in this figure.

For the shown heat sink under free convection or when the pressure drop is not a matter of importance, the rate of entropy generation may be expressed as [21]:

$$
\dot{S}_{g e n}=\frac{Q \theta_{b}}{T_{\infty}^{2}}
$$

where $\theta_{b}=T_{b}-T_{\infty}$, is the difference between heat sink base temperature and ambient temperature. Knowing $Q=\theta_{b} / R_{\text {sink }}$

$$
\dot{S}_{g e n}=\frac{Q^{2} R_{\operatorname{sink}}}{T_{\infty}^{2}}
$$

and the heat sink thermal resistance is defined as: 


$$
R_{\text {sink }}=\frac{1}{\frac{N}{R_{f i n}}+h(N-1) b L}+\frac{t_{b}}{k L W}
$$

in which the second term is heat sink base thermal resistance. It is clear that this resistance is importance when the area of the heating source and heat sink are not the same and no minimum value can be found for this resistance. So, during the optimization procedure, it can be omitted without loss of generality. $R_{\text {fin }}$ is fin thermal resistance and defined as [22]:

$$
\begin{gathered}
R_{f i n}=\frac{1}{\sqrt{h \mathrm{P} k A_{c}} \tanh (m H)} \\
m=\sqrt{\frac{2 h}{k t}}
\end{gathered}
$$

where $\mathrm{P}$ and $\mathrm{A}_{\mathrm{c}}$ are perimeter and cross section area of fin:

$$
\begin{gathered}
\mathrm{P}=2(L+t) \approx 2 \mathrm{~L} \\
A_{c}=L . t
\end{gathered}
$$

So, the dimensionless form of Eq. (4) may be proposed as:

$$
R_{f i n}=\frac{1}{k L \sqrt{2 \cdot B i \overline{\bar{t}}} \tanh \left(\sqrt{2 \cdot B i \frac{\bar{H}^{2}}{\bar{t} \bar{b}}}\right)}
$$

in which over bar means dimensionless form of parameter i.e. $\overline{\mathrm{t}}=\frac{\mathrm{t}}{\mathrm{L}}$ or $\overline{\mathrm{b}}=\frac{\mathrm{b}}{\mathrm{L}}$.

$$
B i=\frac{N u k_{f}}{k}=\frac{h b}{k}
$$

and finally:

$$
R_{\text {sink }}=\frac{1}{k L\left[N \sqrt{2 \cdot B i \overline{\bar{t}}} \tanh \left(\sqrt{\left.2 \cdot B i \frac{\bar{H}^{2}}{\bar{t} \bar{b}}\right)+B i(N-1)}\right]\right.}
$$

and the rate of entropy generation may be redefined as:

$$
\dot{S}_{g e n}=\frac{Q^{2}}{T_{\infty}^{2} k L} \frac{1}{\left[N \sqrt{2 \cdot B i \frac{\bar{t}}{\bar{b}}} \tanh \left(\sqrt{\left.2 \cdot B i \frac{\bar{H}^{2}}{\bar{t} \bar{b}}\right)+B i(N-1)}\right]\right.}
$$

In this regard, the number of entropy generation can be proposed as:

$$
N_{S}=\frac{\dot{S}_{g e n}}{\frac{Q}{T_{\infty} k L} \cdot \frac{Q}{T_{\infty}}}=\frac{1}{N \sqrt{2 \cdot B i \frac{\bar{t}}{\bar{b}}} \tanh \left(\sqrt{2 \cdot B i \frac{\bar{H}^{2}}{\bar{t} \bar{b}}}\right)+B i(N-1)}
$$

Based on heat sink geometry, one can present:

or in the dimensionless form:

$$
N \cdot t+(N-1) \cdot b=W
$$

$$
N \cdot \bar{t}+(N-1) \cdot \bar{b}=\bar{W}
$$




$$
\begin{gathered}
N=\frac{\bar{W}+\bar{b}}{\bar{t}+\bar{b}} \\
N-1=\frac{\bar{W}-\bar{t}}{\bar{t}+\bar{b}}
\end{gathered}
$$

By replacing Eq. (15) and Eq. (16) in Eq. (12), the final form of the number of entropy generation will be:

$$
N_{S}=\frac{1}{\left[\frac{\bar{W}+\bar{b}}{\bar{t}+\bar{b}}\right] \sqrt{2 \cdot B i \frac{\bar{t}}{\bar{b}}} \tanh \left(\sqrt{2 \cdot B i \overline{\bar{H}^{2}} \overline{\bar{b}}}\right)+B i\left(\frac{\bar{W}-\bar{t}}{\bar{t}+\bar{b}}\right)}
$$

To optimize the heat sink, it is just enough to minimize the number of entropy generation, Eq. (17). The effective variables in Eq. (17) are $\mathrm{Bi}, \overline{\mathrm{H}}, \overline{\mathrm{W}}, \overline{\mathrm{t}}$ and $\overline{\mathrm{b}}$. However, considering the arrangement of variables in Eq. (17), it is clear that the number of variables can be reduced more by dividing geometrical parameters by $\bar{b}$. In this way, Eq. (17) may be summarized as follows:

$$
N_{S}=\frac{1}{\left[\frac{\widetilde{W}+1}{\tilde{t}+1}\right] \sqrt{2 . B i \cdot \tilde{t}} \tanh \left(\sqrt{2 . B i \frac{\widetilde{H}^{2}}{\tilde{t}}}\right)+B i\left(\frac{\widetilde{W}-\tilde{t}}{\tilde{t}+1}\right)}
$$

where:

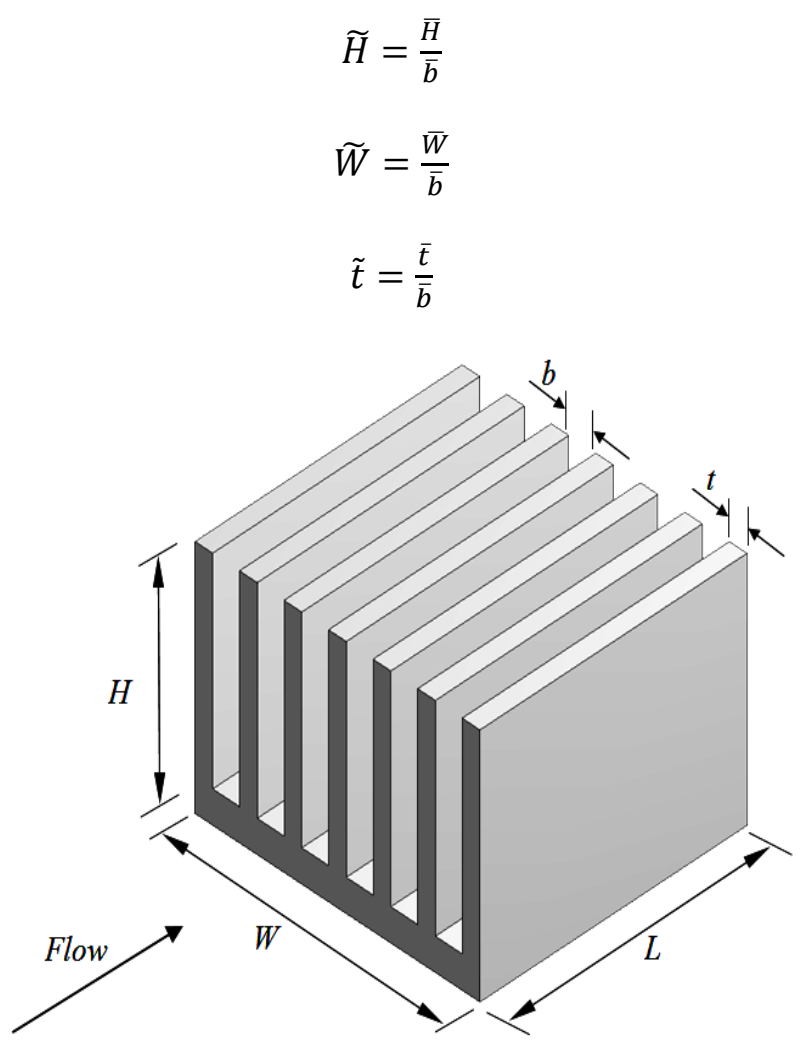

Fig. 1. A typical plate fin heat sink.

\section{Parametric Study}

To study the effect of different variables on the number of entropy generation, more than 200 different cases in a wide range of working condition and geometrical parameters were simulated. In the following parts, the effect of each variable is presented, using Eq. (18). 


\subsection{Effect of $\widetilde{\mathbf{H}}$ on Number of Entropy Generation}

Figure 2 shows variation of number of entropy generation with $\widetilde{\mathrm{H}}$. By increasing $\mathrm{Bi}, \mathrm{N}_{\mathrm{s}}$ approaches to an asymptotic value. By increasing $\widetilde{\mathrm{H}}$, fin surface temperature approaches to ambient temperature. So, depending on $\mathrm{Bi}$, no considerable heat transfer occurs from a longer fins and therefore, increasing the fin length does not affect number of entropy generation.

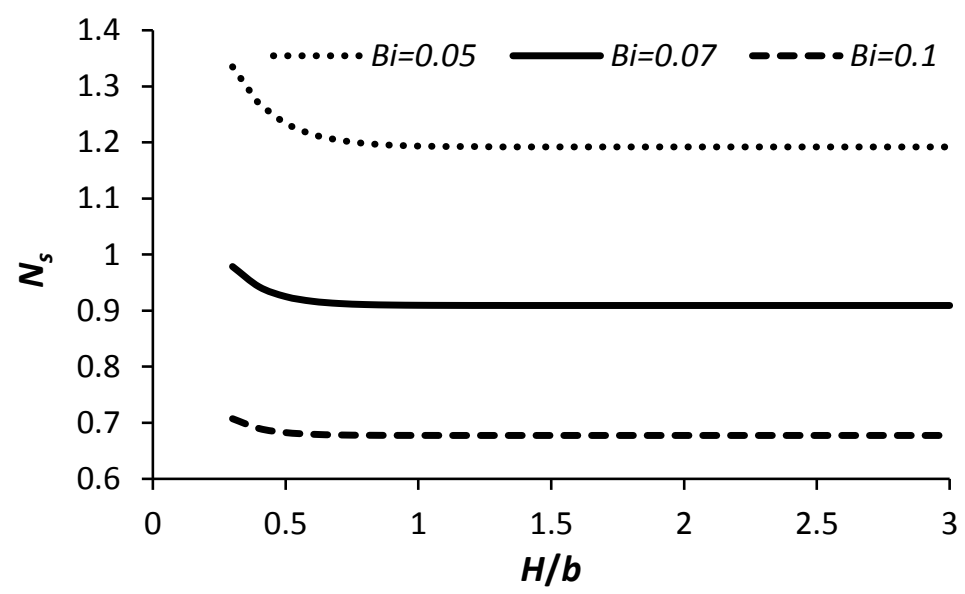

Fig. 2. Variation of number of entropy generation with $\widetilde{H}$.

\subsection{Effect of $\widetilde{\mathbf{W}}$ on Number of Entropy Generation}

Figure 3 shows variation of number of entropy generation with $\widetilde{W}$. Increasing $\widetilde{W}$ results in decreasing number of entropy generation, monotonically. Since by increasing the base heat sink dimension, $\widetilde{W}$, the fin temperature decreases and therefore number of entropy generation decrease as a result.

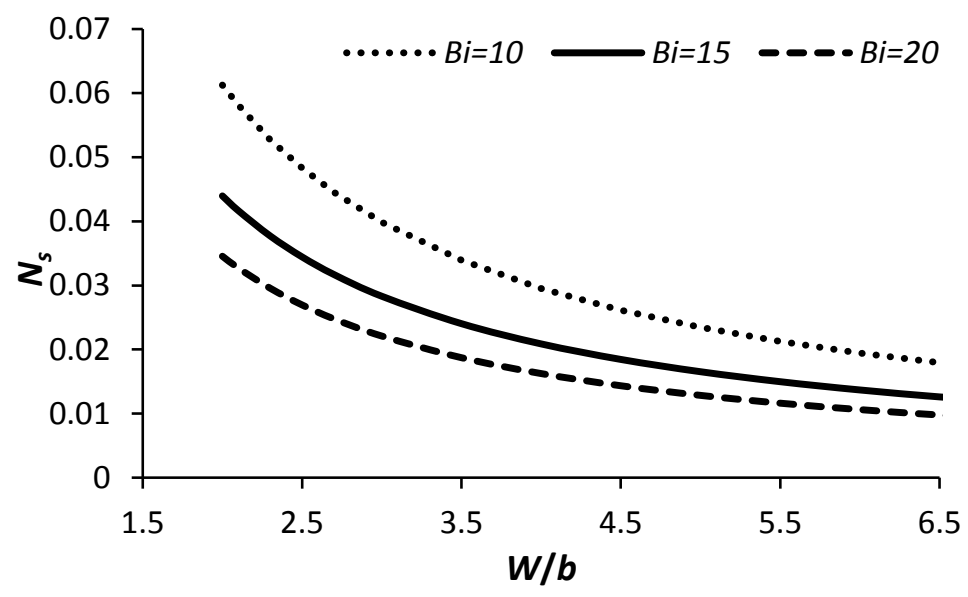

Fig. 3. Variation of number of entropy generation with $\widetilde{W}$.

\subsection{Effect of Bi on Number of Entropy Generation}

The effect of Bi on number of entropy generation is shown in Fig. 4. By increasing Bi the number of entropy generation decreases monotonically. Since in higher $\mathrm{Bi}$, heat transfer occurs at lower temperatures. It is important to note that by increasing $\mathrm{Bi}$, its effect on the number of entropy generation decreases. It is because of this fact that at higher $\mathrm{Bi}$, the conductive resistance of fins gets critical. Moreover, this figure shows that $\widetilde{W}$ does not affect $\mathrm{N}_{\mathrm{s}}$ significantly and for $\widetilde{\mathrm{W}}>2$, its effect cam be neglected. 


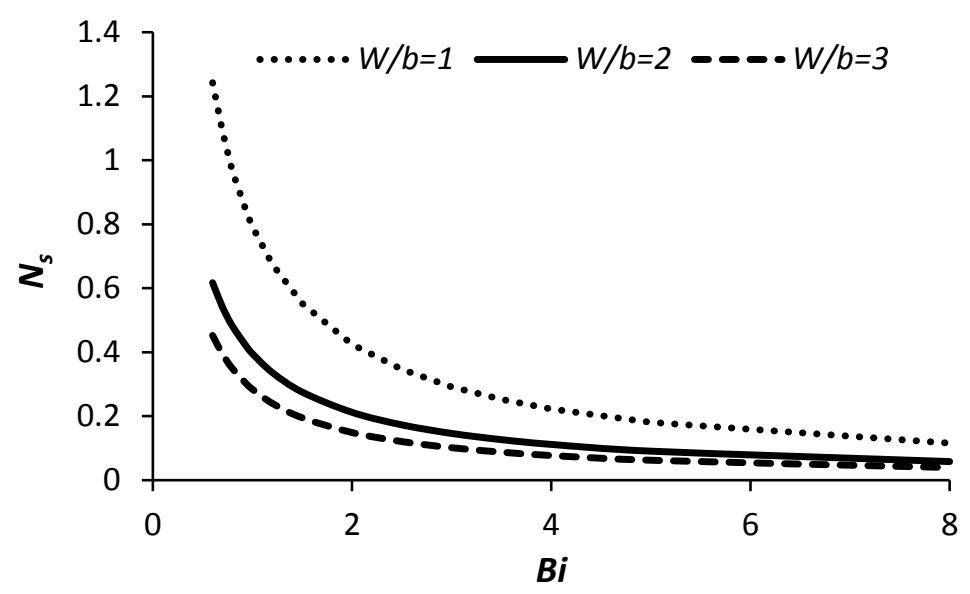

Fig. 4. Variation of number of entropy generation with $B i$.

\subsection{Effect of $\tilde{\mathbf{t}}$ on Number of Entropy Generation}

Figure 5 shows variation of number of entropy generation with $\tilde{\mathrm{t}}$ for $\widetilde{\mathrm{W}}=10$ and $\widetilde{\mathrm{H}}=2$. Based on this figure, by increasing $\tilde{t}, N_{S}$ decreases rapidly to a minimum value and then increases slowly. So, if for any reason, the optimum fin thickness is not accessible, it is just enough to be ensured that the thickness is more that the optimum value. Curves are plotted for three different Bi's and the minimum values are connected to each others. In this regard, the optimum value of $\tilde{\mathrm{t}}$ depends on $\mathrm{Bi}$ also. The other point is that in higher values, the sensitivity of $\mathrm{N}_{s}$ to $\tilde{\mathrm{t}}$ is lower. This, may be interpreted in this way that by increasing the fin thickness, the thermal conductive resistance decreases but on the other hand, increasing the fin thickness in the heat sink with constant width decreases the possible number of fins as well as total heat transfer area. Therefore, for a specified heat generation rate, higher temperature difference is required which means higher entropy generation rate. It is clear that in lower $\mathrm{Bi}$, the role of convective heat transfer is weak, therefore the decrease of fin conductive thermal resistance in higher fin thickness is less effective.

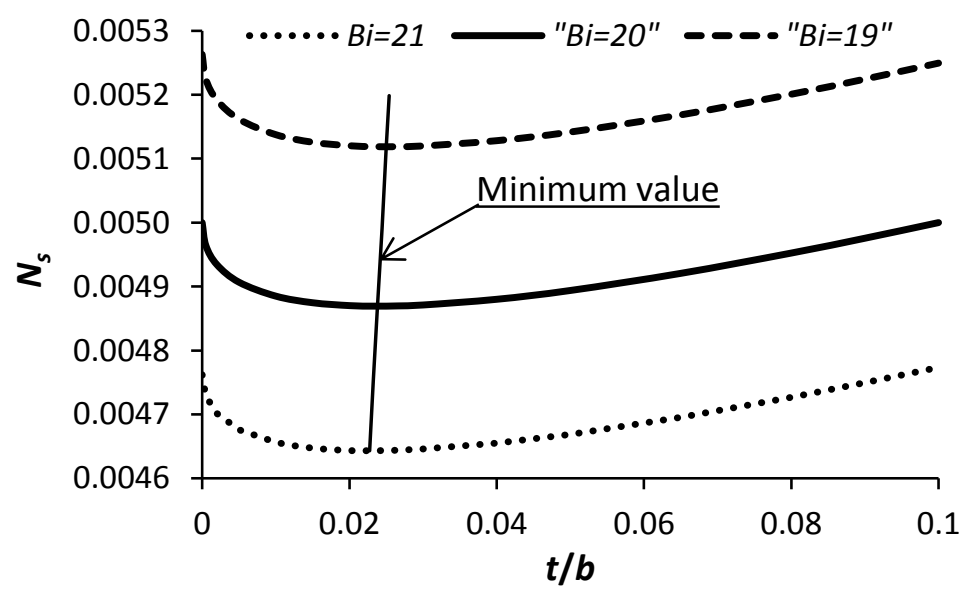

Fig. 5. Variation of number of entropy generation with $\tilde{t}$.

\section{Geometry Optimization}

Based on the performed parametric study, increasing all the variables with the exception of $\tilde{t}$, results in decreasing $\mathrm{N}_{\mathrm{s}}$. So, actually no optimum value may be found. But for the variable $\tilde{\mathrm{t}}$, there is an optimum value. To find the optimum value of $\tilde{\mathfrak{t}}$, the denominator of Eq. (18) must be maximized or: 


$$
\frac{d}{d \tilde{t}}\left\{\left[\frac{\widetilde{W}+1}{\tilde{t}+1}\right] \sqrt{2 \cdot B i \cdot \tilde{t}} \tanh \left(\sqrt{2 \cdot B i \frac{\widetilde{H}^{2}}{\tilde{t}}}\right)+B i\left(\frac{\widetilde{W}-\tilde{t}}{\tilde{t}+1}\right)\right\}=0
$$

However, as it was shown, the value of $\widetilde{W}$ on optimum value of $\tilde{\mathrm{t}}$ is actually effectless. Eq. (22) was solved numerically by iterative method [23] for a large variety of $\widetilde{\mathrm{H}}$ 'Bi and $\widetilde{\mathrm{W}}$. Some results are presented in Table 1. As it can be observed, changing the value of $\widetilde{W}$ from 2 to 500 does not affect the optimum dimensionless fin thickness. Owing to this fact, Eq. (22) may be approximate as:

$$
\frac{d}{d \tilde{t}}\left\{\left[\frac{\sqrt{2 . B i \cdot \tilde{t}}}{\tilde{t}+1}\right] \tanh \left(\sqrt{2 \cdot B i \frac{\widetilde{H}^{2}}{\tilde{t}}}\right)+\left(\frac{B i}{\tilde{t}+1}\right)\right\}=0
$$

and the solution will be:

$$
\frac{\tanh \left(\sqrt{2 \cdot B i \frac{\widetilde{H}^{2}}{\tilde{t}}}\right) \cdot B i}{(\tilde{t}+1) \sqrt{2 \cdot B i \cdot \tilde{t}}}-\frac{\sqrt{2 \cdot B i \cdot \tilde{t}}}{(\tilde{t}+1)^{2}} \tanh \left(\sqrt{2 \cdot B i \frac{\widetilde{H}^{2}}{\tilde{t}}}\right)-\frac{B i \cdot \widetilde{H}}{\tilde{t}+1} \frac{1-\left[\tanh \left(\sqrt{2 \cdot B i \frac{\widetilde{H}^{2}}{\tilde{t}}}\right)\right]^{2}}{\tilde{t}}-\frac{B i}{(\tilde{t}+1)^{2}}=0
$$

\begin{tabular}{|c|c|c|c|}
\hline$\widetilde{\boldsymbol{H}}$ & $B i$ & $\widetilde{W}$ & $\tilde{\boldsymbol{t}}_{\boldsymbol{o p t}}$ \\
\hline \multirow{15}{*}{1} & \multirow{5}{*}{1} & 2 & 0.246 \\
\hline & & 5 & 0.246 \\
\hline & & 10 & 0.246 \\
\hline & & 100 & 0.246 \\
\hline & & 500 & 0.246 \\
\hline & \multirow{5}{*}{5} & 2 & 0.084 \\
\hline & & 5 & 0.084 \\
\hline & & 10 & 0.084 \\
\hline & & 100 & 0.084 \\
\hline & & 500 & 0.084 \\
\hline & \multirow{5}{*}{15} & 2 & 0.031 \\
\hline & & 5 & 0.031 \\
\hline & & 10 & 0.031 \\
\hline & & 100 & 0.031 \\
\hline & & 500 & 0.031 \\
\hline
\end{tabular}

\begin{tabular}{|c|c|c|c|}
\hline$\widetilde{\boldsymbol{H}}$ & $B i$ & $\widetilde{W}$ & $\tilde{\boldsymbol{t}}_{\boldsymbol{o p t}}$ \\
\hline \multirow{15}{*}{5} & \multirow{5}{*}{1} & 2 & 0.268 \\
\hline & & 5 & 0.268 \\
\hline & & 10 & 0.268 \\
\hline & & 100 & 0.268 \\
\hline & & 500 & 0.268 \\
\hline & \multirow{5}{*}{5} & 2 & 0.084 \\
\hline & & 5 & 0.084 \\
\hline & & 10 & 0.084 \\
\hline & & 100 & 0.084 \\
\hline & & 500 & 0.084 \\
\hline & \multirow{5}{*}{15} & 2 & 0.031 \\
\hline & & 5 & 0.031 \\
\hline & & 10 & 0.031 \\
\hline & & 100 & 0.031 \\
\hline & & 500 & 0.031 \\
\hline
\end{tabular}

Table 1. Optimum dimensionless fin thickness.

\begin{tabular}{|c|c|c|c|}
\hline$\widetilde{\boldsymbol{H}}$ & $B i$ & $\widetilde{W}$ & $\tilde{\boldsymbol{t}}_{\boldsymbol{o p t}}$ \\
\hline \multirow{15}{*}{20} & \multirow{5}{*}{1} & 2 & 0.268 \\
\hline & & 5 & 0.268 \\
\hline & & 10 & 0.268 \\
\hline & & 100 & 0.268 \\
\hline & & 500 & 0.268 \\
\hline & \multirow{5}{*}{5} & 2 & 0.084 \\
\hline & & 5 & 0.084 \\
\hline & & 10 & 0.084 \\
\hline & & 100 & 0.084 \\
\hline & & 500 & 0.084 \\
\hline & \multirow{5}{*}{15} & 2 & 0.031 \\
\hline & & 5 & 0.031 \\
\hline & & 10 & 0.031 \\
\hline & & 100 & 0.031 \\
\hline & & 500 & 0.031 \\
\hline
\end{tabular}

Unfortunately, no explicit results may be found for Eq. (24). Figure 6 and Figure 7 show the variation of $\tilde{\mathrm{t}}_{\text {opt }}$ as a function of dimensionless group, $\mathrm{Bi} \widetilde{\mathrm{H}}^{2}$ for $\widetilde{\mathrm{H}}=0.2$ and $\widetilde{\mathrm{H}}=1.0$. As it can be observe, for the limiting values of $\mathrm{Bi} \widetilde{\mathrm{H}}^{2}$, the behavior of the curve is basically different. 


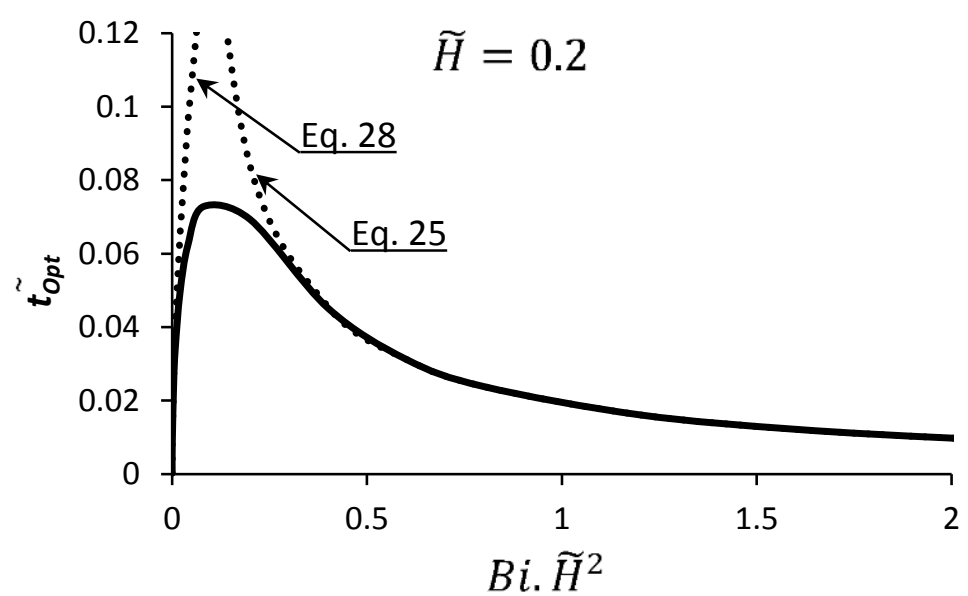

Fig. 6. Variation of $\tilde{t}_{\text {opt }}$ with $B i \widetilde{H}^{2}$ for $\widetilde{H}=0.2$.

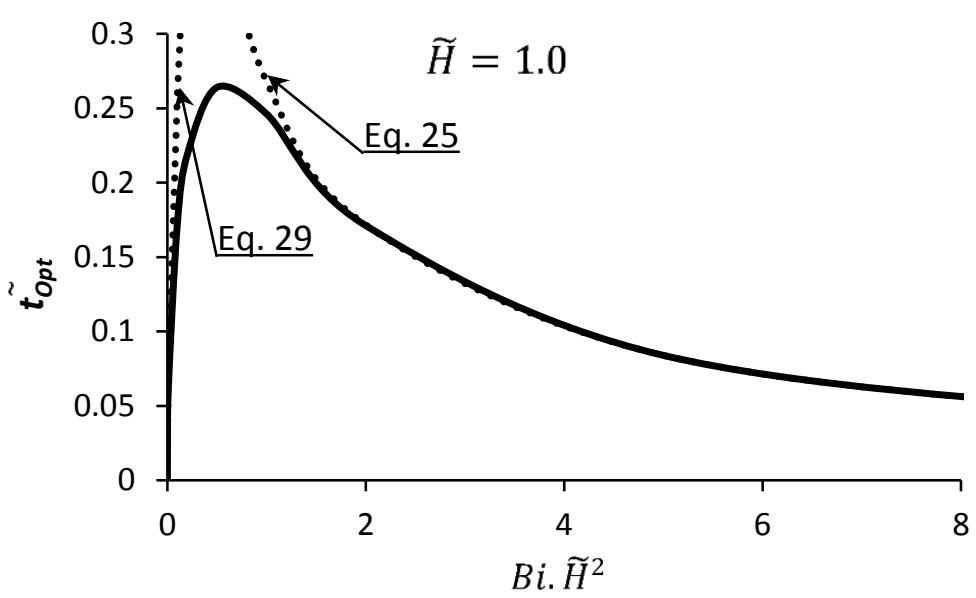

Fig. 7. Variation of $\tilde{t}_{\text {opt }}$ with $B i \widetilde{H}^{2}$ for $\widetilde{H}=1.0$.

So, in the following, the optimum values of $\tilde{\mathrm{t}}_{\mathrm{opt}}$ for the limiting values of $\mathrm{Bi} \widetilde{\mathrm{H}}^{2}$ are studied.

\subsection{Limiting Case in which Bi. $\widetilde{\mathbf{H}}^{2} \rightarrow \infty$}

For this case:

$$
\lim _{B i \cdot \widetilde{H}^{2} \rightarrow \infty} \tanh \left(\sqrt{2 \cdot B i \frac{\widetilde{H}^{2}}{\tilde{t}}}\right)=1
$$

and the result of Eq. (24) is:

$$
\tilde{t}_{\infty}=(1+B i)-\sqrt{B i^{2}+2 B i}
$$

\subsection{Limiting Case in which Bi. $\widetilde{\mathbf{H}}^{2} \rightarrow \mathbf{0}$}

For this case $\tanh \left(\sqrt{2 \cdot \mathrm{Bi} \frac{\widetilde{\mathrm{H}}^{2}}{\tilde{\mathrm{t}}}}\right)$ may be approximated by its Taylor series: 


$$
\begin{gathered}
\tanh \left(\sqrt{2 \cdot B i \frac{\widetilde{H}^{2}}{\tilde{t}}}\right) \approx \sqrt{2 \cdot B i \frac{\widetilde{H}^{2}}{\tilde{t}}}-\frac{\sqrt{2 \cdot B i \frac{\widetilde{H}^{2}}{\tilde{t}}}}{3}+O\left(\left[2 \cdot B i \frac{\widetilde{H}^{2}}{\tilde{t}}\right]^{2}\right) \\
{\left[\tanh \left(\sqrt{2 \cdot B i \frac{\widetilde{H}^{2}}{\tilde{t}}}\right)\right]^{2} \approx 2 \cdot B i \frac{\widetilde{H}^{2}}{\tilde{t}}}
\end{gathered}
$$

By this approximation, the result of Eq. (24) is:

$$
\tilde{t}_{\infty}=\frac{2}{3} \frac{\widetilde{H}\left(2 \cdot B i \cdot \widetilde{H}^{2}+\sqrt{B i . \widetilde{H}^{2}\left(6+4 . B i \cdot \widetilde{H}^{2}\right)+3 B i \cdot \widetilde{H}}\right)}{2 \widetilde{H}+1}
$$

Eq. (22) was solved numerically for 220 different cases by means of Newton-Raphson method [23] and by using blending technique [24], the following equation was derived:

$$
\tilde{t}_{O p t}=\left(\tilde{t}_{\infty}^{-1}+\tilde{t}_{0}^{-1}\right)^{-1}
$$

The comparison of Eq. (30) against numerical results is shown in Fig. 8 which shows a good agreement.

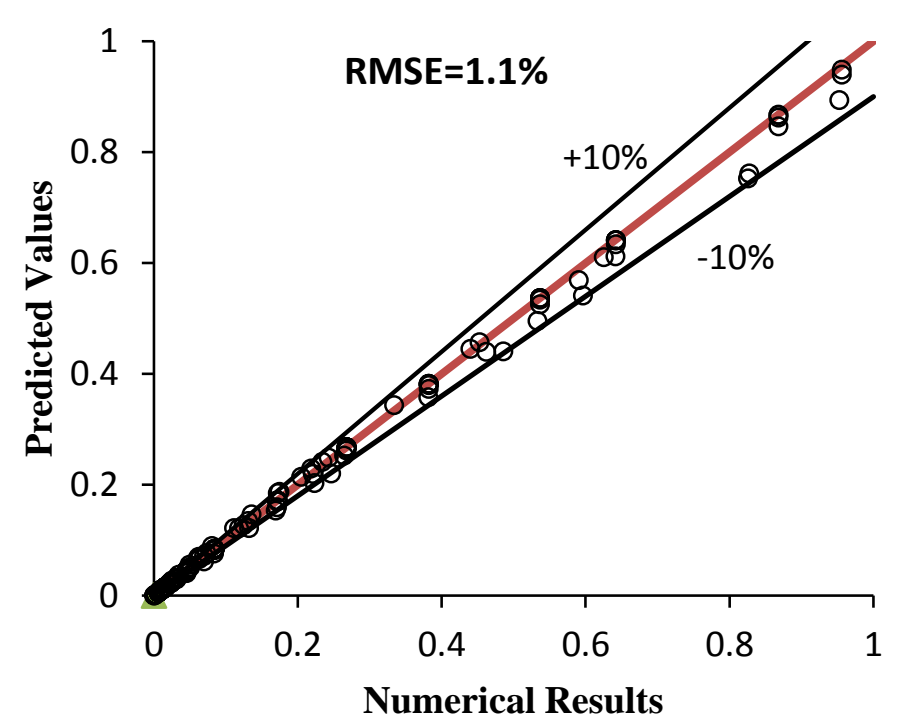

Fig. 8. Comparison of predicted values against numerical results.

So, an explicit expression is proposed for optimum dimensionless thickness as a function of $\widetilde{\mathrm{H}}$ and $\mathrm{Bi}$. In the following the way of using Eq. (30) will be illustrated by means of several examples.

\section{Heat Sink Optimization under Natural Convection}

The $\mathrm{Nu}$ for a plate fin heat sink in natural convection may be presented as follows [25]:

$$
N u=\frac{h b}{k_{f}}=\left(\frac{576}{\left(\eta_{f i n} E l\right)^{2}}+\frac{2.873}{\left(\eta_{f i n} E l\right)^{0.5}}\right)^{-\frac{1}{2}}
$$

in which

$$
E l=R a \cdot b^{4} / L^{4}=\frac{g \beta \theta_{b} P r b^{4}}{L v^{2}}=\frac{g \beta \theta_{b} b^{4}}{L v \alpha}
$$




$$
\eta_{\text {fin }}=\tanh (m H) / m H
$$

and $m$ can be calculated using Eq. (5).

$>$ Example I: Optimizing fin thickness (1 geometrical parameter)

As a 1 st example, consider a typical plate fin heat sink. Its geometrical parameters are specified in Table 2.

Table 2. Parameters of a plate fin heat sink $\mathrm{n}$ thickness.

\begin{tabular}{|c|c|c|c|c|c|c|c|c|c|c|c|}
\hline $\begin{array}{c}W \\
(\mathbf{m})\end{array}$ & $\begin{array}{c}\boldsymbol{H} \\
(\mathbf{m})\end{array}$ & $\begin{array}{c}\boldsymbol{L} \\
(\mathbf{m})\end{array}$ & $\begin{array}{c}\boldsymbol{t}_{\boldsymbol{b}} \\
(\mathbf{m})\end{array}$ & $\begin{array}{c}\boldsymbol{k} \\
(\mathbf{W} / \mathbf{m K})\end{array}$ & $\begin{array}{c}\boldsymbol{\theta}_{\boldsymbol{b}} \\
(\mathbf{K})\end{array}$ & $\begin{array}{c}\boldsymbol{T}_{\text {amb }} \\
(\mathbf{K})\end{array}$ & $\begin{array}{c}\mathbf{b} \\
(\mathbf{1} / \mathbf{K})\end{array}$ & $\begin{array}{c}\mathbf{n} \\
\left(\mathbf{m}^{2} / \mathbf{s}\right)\end{array}$ & $\boldsymbol{P r}$ & $\boldsymbol{R a}$ & $\begin{array}{c}\boldsymbol{k}_{\text {air }} \\
(\mathbf{W} / \mathbf{m K})\end{array}$ \\
\hline 0.1 & 0.05 & 0.1 & 0.0015 & 200 & 55 & 300 & 0.0033 & $1.59 \mathrm{E}-05$ & 0.707 & $5.03 \mathrm{E}+06$ & $2.63 \mathrm{E}-02$ \\
\hline
\end{tabular}

Equationbs (30), (31) and (3) can be used to find the optimum thickness. The aforementioned equations are rearranged in the following:

$$
\begin{gathered}
\frac{b}{t}=\frac{3}{2} \frac{2 \frac{H}{b}+1}{\left(\frac{H}{b}\right)\left(2 \cdot B i \cdot\left(\frac{H}{b}\right)^{2}+\sqrt{B i \cdot\left(\frac{H}{b}\right)^{2}\left(6+4 \cdot B i \cdot\left(\frac{H}{b}\right)^{2}\right)+3 B i \cdot \frac{H}{b}}\right)}+\frac{1}{(1+B i)-\sqrt{B i^{2}+2 B i}} \\
B i \frac{k}{k_{f}}=\left[\frac{576}{\left.\left(\frac{g \cdot \beta \cdot P r \cdot b^{4}}{v^{2} \cdot L} \theta_{b} \frac{\tanh \left(\sqrt{2 \cdot B i \frac{H^{2}}{b . t}}\right)}{\sqrt{2 \cdot B i \frac{H^{2}}{b \cdot t}}}\right)^{2}+\frac{2 \cdot 873}{\left(\frac{g \cdot \beta \cdot P r \cdot b^{4}}{v^{2} \cdot L} \theta_{b} \frac{\tanh \left(\sqrt{2 \cdot B i \frac{H^{2}}{b . t}}\right)}{\sqrt{2 \cdot B i \frac{H^{2}}{b . t}}}\right)^{0.5}}\right]^{-\frac{1}{2}}}\right. \\
\frac{\theta_{b}}{Q}=\frac{1}{k \cdot L\left[\frac{W+b}{t+b}\right] \sqrt{2 \cdot B i \frac{t}{b}} \tanh \left(\sqrt{2 \cdot B i \frac{H^{2}}{b \cdot t}}\right)+B i\left(\frac{W-t}{t+b}\right)}+\frac{t_{b}}{k \cdot L \cdot W}
\end{gathered}
$$

This system of equations involves three unknown variables i.e. $\mathrm{t}, \mathrm{b}$ and $\mathrm{Bi}$. The optimum thickness is found to be $t_{\mathrm{opt}}=0.0005 \mathrm{~m}$ and the number of fins may be found according to Eq. (15).

$$
N=\frac{W+b}{t+b}=\frac{0.1+0.006}{0.0005+0.006}=16.31 \approx 16
$$

The variation of heat transfer rate with number of fins are illustrated in Fig. 9 which shows good agreement with the found solution, $\mathrm{N}=16$.

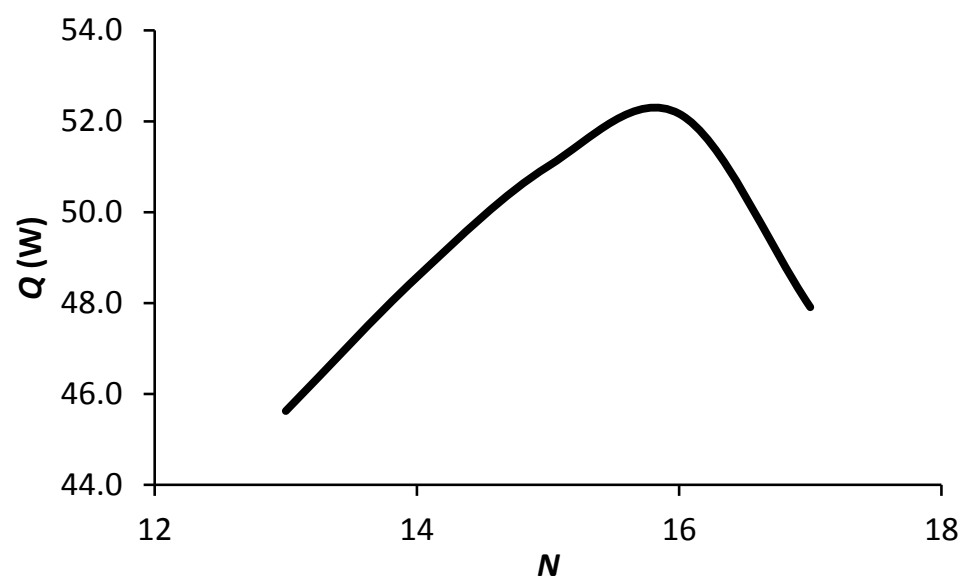


Fig. 9. Variation of heat transfer rate with number of fins.

Example II: Optimizing fin height and spacing (2 geometrical parameters)

For the previous heat sink, it is now assumed that required heat generation rate and fin thickness are specified i.e. $\mathrm{Q}=40 \mathrm{~W}$ and $\mathrm{t}=3 \mathrm{~mm}$. So, the optimum values are:

$$
H_{\text {opt }}=0.125 \mathrm{~m} \quad b_{\text {opt }}=0.028 \mathrm{~m} \quad B i=0.00098
$$

$>$ Example III: Optimizing fin thickness and spacing (2 geometrical parameters)

This time, it is assumed that for the aforementioned heat sink, for the specified heat generation rate, the maximum working temperature is limited to $55 \mathrm{~K}$. The optimum values are:

$$
t_{\text {opt }}=0.0018 \mathrm{~m} \quad b_{\text {opt }}=0.01 \mathrm{~m} \quad B i=0.00037
$$

\section{Heat Sink Optimization under Forced Convection}

Because no specific assumption is made for $\mathrm{Nu}$, Eq. (30) is still applicable for forced convection whenever the pressure drop is not a matter of importance (heat sinks of all small electronic devices such as CPU, may be included in this category). The $\mathrm{Nu}$ for a plate fin heat sink in forced convection may be presented as follows [26]:

$$
\begin{gathered}
N u=\frac{h b}{k_{f}}=\left[\left(\frac{R e_{b}^{*} P r}{2}\right)^{-3}+\left(0.664 \sqrt{\operatorname{Re}_{b}^{*}} \operatorname{Pr}^{\frac{1}{3}} \sqrt{1+\frac{3.65}{\sqrt{R e_{b}^{*}}}}\right)^{-3}\right]^{-\frac{1}{3}} \\
\operatorname{Re}_{b}^{*}=\operatorname{Re}_{b}\left(\frac{b}{L}\right)=\frac{b \cdot V_{c h}}{v}\left(\frac{b}{L}\right)
\end{gathered}
$$

The total pressure drop of fluid flowing through a plate fin heat sink is [27]:

$$
\Delta P=\left(K_{c}+K_{e}+\frac{f_{a p p} L(2 H+b)}{b H}\right) \frac{1}{2} \rho V_{c h}^{2}
$$

Chanel velocity is related to free stream velocity by:

$$
V_{c h}=V_{f}\left(1+\frac{t}{b}\right)
$$

$\mathrm{K}_{\mathrm{c}}$ and $\mathrm{K}_{\mathrm{e}}$ are sudden contraction and expansion pressure drop coefficient as defined as [28]:

$$
\begin{gathered}
K_{c}=0.42\left(1+\sigma^{2}\right) \\
K_{e}=\left(1-\sigma^{2}\right)^{2}
\end{gathered}
$$

and $\sigma$ is the plate fin heat sink porosity,

$$
\sigma=\frac{1}{\left(1+\frac{t}{b}\right)}
$$

Apparent friction factor in Eq. (40) is defined as following [29]: 


$$
\begin{gathered}
f_{a p p} R e_{c h}=\left[\left(\frac{3.44}{\sqrt{L_{c h}^{*}}}\right)^{2}+\left(f R e_{c h}\right)^{2}\right]^{\frac{1}{2}} \\
L_{c h}^{*}=\frac{L}{D_{c h} R e_{c h}} \\
R e_{c h}=\frac{\rho V_{c h} D_{c h}}{\mu} \\
D_{c h} \approx 2 b \\
f R e_{c h}=24-32.527\left(\frac{b}{H}\right)+46.721\left(\frac{b}{H}\right)^{2}-40.829\left(\frac{b}{H}\right)^{3}+22.954\left(\frac{b}{H}\right)^{4}-6.089\left(\frac{b}{H}\right)^{5}
\end{gathered}
$$

$>$ Example IV: Optimizing fin thickness (1 geometrical parameter)

As an example, consider again the plate fin heat sink specified in Example I and Table 2 but under forced convection. The air velocity exiting the fan is assumed $2 \mathrm{~m} / \mathrm{s}$. Accordingly, the optimum thickness is $\mathrm{t}_{\mathrm{opt}}=$ $0.001 \mathrm{~m}$ and the number of fins is:

$$
N=\frac{W+b}{t+b}=\frac{0.1+0.006}{0.001+0.006}=15.1 \approx 15
$$

The variation of heat transfer rate with number of fins is illustrated in Fig. 10 and shows good agreement with the found solution, $\mathrm{N}=15$.

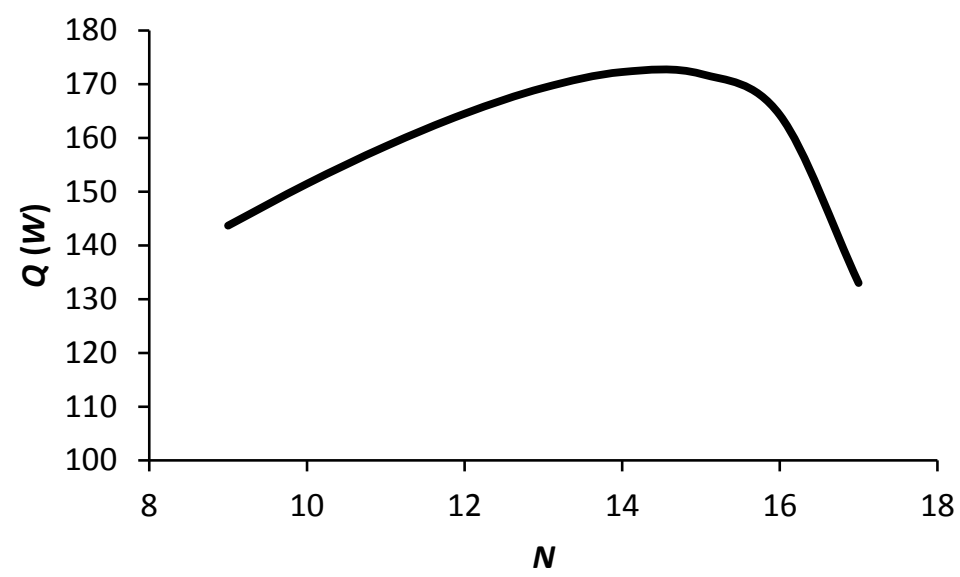

Fig. 10. Variation of heat transfer rate with number of fins.

This problem is repeated for other velocities and results are illustrated in Fig. 11. 


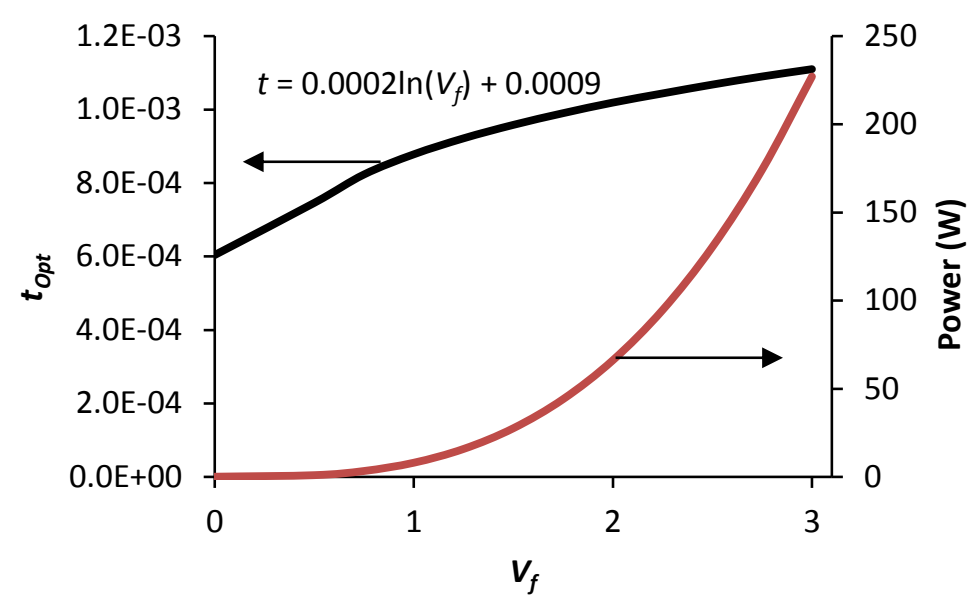

Fig. 11. Variation of $t_{o p t}$ and power consumption with $V_{f}$.

$>$ Example V: Optimizing fin height and spacing (2 geometrical parameters)

It is now assumed that required heat generation rate and fin thickness are specified and i.e. $Q=40 \mathrm{~W}$ and $\mathrm{t}=3 \mathrm{~mm}$. So, the optimum values are:

$$
H_{\text {opt }}=0.035 \mathrm{~m} \quad b_{\text {opt }}=0.015 \mathrm{~m} \quad B i=0.0015
$$

which shows that shorter fin with larger fin spacing is required in comparison with natural convection.

Example VI: Optimizing fin number using fan curve

In Example IV, air approach velocity was specified at first. However, it is clear that this velocity depends on the type of fan as well as pressure drop. So, it is more realistic to specify fan curve, instead. Fig. 12 shows the inverse relation between pressure drop and approach velocity for ETRI-DC-373DH fan. The pressure drop for various numbers of fins is also shown in this figure. The intersections between these curves show the system operating points.

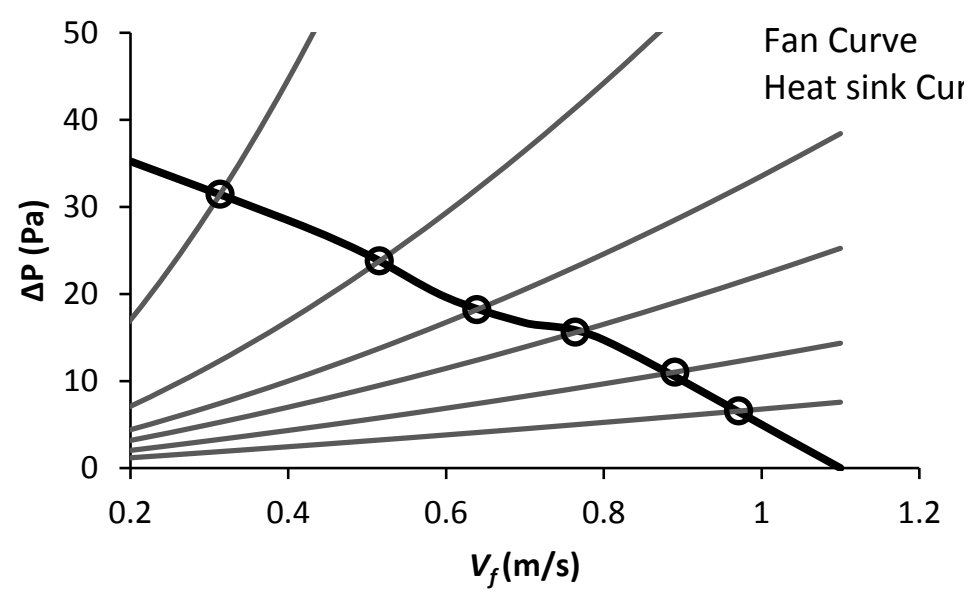

Fig. 12. Operating points for various fin thickness.

The following simple curve can be fitted to the locus of operating points:

$$
V_{f}=\frac{1}{0.9515+121.31 t}
$$


Knowing the approach velocity as a function of fin thickness, the following optimized results are obtained:

$$
V_{f}=0.994 \mathrm{~m} / \mathrm{s} \quad \Delta P=5.3 \mathrm{~Pa} \quad t_{\text {opt }}=0.00045 \mathrm{~m} \quad Q=252.7 \mathrm{~W}
$$

\section{Conclusions}

In the present study, by using the entropy generation minimization method, a general equation was proposed to relate the dimensionless parameters of the plate fin heat sink in optimum working condition. The importance of this work is that it has been based on dimensionless parameters. So the results are not limited to a specific geometry or some special examples. Moreover, since no restricting assumption was made, this equation can be used for both natural and forced convection. Six examples were employed to clear the method of using the proposed equation. Although the manufacturing process of a heat sink is always accompanied with many different restrictions such as machining tools, those examples showed that this method had enough flexibility to consider all these restrictions. It was shown also that how to use a fan curve instead of approach velocity in optimizing heat sink. Although considering fan curve is a little more difficult in comparison, it must be kept in mind that it is more realistic. Because the volumetric flow rate as well as approach velocity in a fan are also, functions of pressure drop and it is only a simplification to evaluate the flow rate independence of pressure drop.

\section{Acknowledgments}

The author would like to thank Islamic Azad University, Marvdasht branch for supporting this research project.

\section{Nomenclature}

$\begin{array}{lll}A_{c}=L . t & \left(\mathrm{~m}^{2}\right) & \text { Cross section area of fin } \\ b & (\mathrm{~m}) & \text { Fin spacing } \\ \bar{b}=\frac{b}{L} & --- & \text { Dimensionless Fin spacing } \\ B i=\frac{N u k_{f}}{k}=\frac{h b}{k} & --- & \text { Biot } \text { Number } \\ E l & -- & \text { Elenbass } \text { Number } \\ f_{a p p} & --- & \text { Apparent friction factor } \\ H & (\mathrm{~m}) & \text { Fin height } \\ \bar{H}=\frac{H}{L} & --- & \text { Dimensionless fin height } \\ H & (\mathrm{~W} / \mathrm{m} 2 \mathrm{~K}) & \text { Convection heat transfer coefficient } \\ K_{c} & --- & \text { Sudden contraction pressure drop } \\ K_{e} & --- & \text { coefficient } \\ k & (\mathrm{~W} / \mathrm{mK}) & \text { Sudden expansion pressure drop coefficient } \\ k_{f} & (\mathrm{~W} / \mathrm{mK}) & \text { Heat sink thermal conductivity } \\ L & (\mathrm{~m}) & \text { Fluid thermal conductivity } \\ N & --- & \text { Heat sink Length } \\ N u=\frac{h b}{k_{f}} & --- & \text { Fin Number } \\ \mathrm{P}=2(L+t) \approx 2 \mathrm{~L} & \left(\mathrm{~m}{ }^{2}\right) & \text { Nusselt } \text { Number } \\ Q & (\mathrm{~W}) & \text { Perimeter of fin } \\ R_{f i n} & (\mathrm{~K} / \mathrm{W}) & \text { Heat transfer rate } \\ R_{\text {sink }} & (\mathrm{K} / \mathrm{W}) & \text { Fin thermal resistance } \\ \dot{S}_{g e n} & (\mathrm{~W} / \mathrm{K}) & \text { Heat sink thermal resistance } \\ T_{\infty} & (\mathrm{K}) & \text { Rate of entropy generation } \\ T_{b} & (\mathrm{~K}) & \text { Ambient temperature } \\ & & \text { Heat sink base temperature } \\ & & \end{array}$




$\begin{array}{lll}t & (\mathrm{~m}) & \text { Fin thickness } \\ t_{b} & (\mathrm{~m}) & \text { Heat sink base thickness } \\ t_{\text {opt }} & (\mathrm{m}) & \text { Optimum fin thickness } \\ \bar{t}=\frac{t}{L} & --- & \text { Dimensionless thickness } \\ V_{c h} & (\mathrm{~m} / \mathrm{s}) & \text { Channel velocity } \\ V_{f} & (\mathrm{~m} / \mathrm{s}) & \text { Free stream velocity } \\ W & (\mathrm{~m}) & \text { Heat sink width } \\ \bar{W}=\frac{W}{L} & --- & \text { Dimensionless heat sink width } \\ \eta_{f i n} & --- & \text { Fin efficiency } \\ \theta_{b}=T_{b}-T_{\infty} & (\mathrm{K}) & \text { Temperature difference } \\ \sigma & --- & \text { Heat sink porosity }\end{array}$

\section{References}

[1] P. Teertstra, M. M. Yovanovich, and J. R. Culham, "Analytical forced convection modeling of plate fin heat sinks," in Semiconductor Thermal Measurement and Management Symposium, Fifteenth Annual IEEE, 1999, pp. 34-41.

[2] S. Y. Kim and R. L. Webb, "Analysis of convective thermal resistance in ducted fan heat sinks," IEEE Transactions on Components and Packaging Technologies, vol. 29, pp. 439-448, 2006.

[3] C. J. Kobus and T. Oshio, "Development of a theoretical model for predicting the thermal performance characteristics of a vertical pin-fin array heat sink under combined forced and natural convection with impinging flow," Int. J. Heat Mass Transfer, vol. 48, pp. 1053-1063, 2005.

[4] S. Chingulpitak, N. Chimresa, K. Nilpueng, and S. Wongwises, "Experimental and numerical investigations of heat transfer and flow characteristics of cross-cut heat sinks," Int. J. Heat Mass Transfer, vol. 102, pp. 145-153, 2016.

[5] J. Hoa, K. Wonga, K. Leonga, and T. Wong, "Convective heat transfer performance of airfoil heat sinks fabricated by selective laser melting," Int.J. Therm. Sci., vol. 114, pp. 213-228, 2017.

[6] S. Huanga, J. Zhaob, L. Gong, and X. Duana, "Thermal performance and structure optimization for slotted microchannel heat sink," Appl. Therm. Eng., vol. 115, pp. 1266-1276, 2017.

[7] S. B. Sathe, "An analytical study of the optimized performance of an impingement heat sink," J. Electron. Packaging, vol. 126, pp. 528-534, 2004.

[8] O. Leon, "Comparison between the standard and staggered layout for cooling fins in forced convection cooling," J. Electron. Packaging, vol. 125, pp. 442-446, 2003.

[9] P. H. Oosthuizen, "A numerical study of mixed convective heat transfer from a parallel fin heat sink," in 38 th ALAA Thermophysics Conference, Toronto, Ontario Canada, AIAA, 2005, p. 4824.

[10] A. Z. Sahin, "Second law analysis of laminar viscous flow through a duct subjected to constant wall temperature," Int. J. Heat Mass Transfer, vol. 120, pp. 76-83, 1998.

[11] S. Mahmud and R. A. Fraser, "The second law analysis in fundamental convective heat transfer problems," Int. J. Therm. Sci., vol. 42, pp. 177-186, 2003.

[12] S. Z. Shuja and B. S. Yilbas, "Flow through a protruding bluff body-heat and irreversibility analysis," Exergy, vol. 1, pp. 209-215, 2001.

[13] A. C. Baytas, "Entropy generation for natural convection in an inclined porous cavity," Int. J. Heat Mass Transfer, vol. 43, pp. 2089-2099, 2000.

[14] I. Dagtekin, H. F. Oztop, and A. Z. Sahin, "An analysis of entropy generation through a circular duct with different shaped longitudinal fins for laminar flow," Int. J. Heat Mass Transfer, vol. 48, pp. 71-181, 2005.

[15] T. Furukawa and W. J. Yang, "Reliability of heat sink optimization using entropy generation minimization," in $8^{\text {th }} A I A A / A S M E$ Joint Thermophysics and Heat Transfer Conference, American Institute of Aeronautics and Astronautics, St. Louis, Missouri, 2002. 
[16] C. J. Shih and G. C. Liu, "Optimal design methodology of plate-fin heat sinks for electronic cooling using entropy generation strategy," IEEE Trans. Compon. Packag. Technol., vol. 27, no. 3, pp. 551-559, 2004.

[17] J. Culham and Y. Muzychka, "Optimization of plate fin heat sinks using entropy generation minimization," IEEE Trans. Compon. Packag. Technol., vol. 24, vol. 159-165, 2001. Available: http://dx.doi.org/10.1109/6144.926378

[18] W. Khan, J. Culham, and M. Yovanovich, "Optimization of pin-fin heat sinks using entropy generation minimization,” IEEE Trans. Compon. Packag. Technol., vo. 28, pp. 247-254, 2005.

[19] W. Khan and J. Culham, "The role of fin geometry in heat sink performance," J. Electron. Packaging, vol. 128, pp. 324-330, 2006.

[20] M. Asadi and A. Shalchi-Tabrizi, "Introducing some correlations to calculate entropy generation in extended surfaces with uniform cross sectional area," Phys. Sci. Int. J., vol. 4, no. 3, pp. 402-415, 2014.

[21] A. Bejan and E. Sciubba, "The optimal spacing of parallel plates cooled by forced convection," Int. J. Heat Mass Transfer, vol. 35, pp. 3259-3264, 1992.

[22] F. P. Incropera and D. P. Dewitt, Fundamentals of Heat and Mass Transfer. New York: Wiley, 1996.

[23] W. F. Stoecker, Design of Thermal Systems. 3rd ed. McGraw Hill, 1989.

[24] S. W. Churchill and R. Usagi, "A general expression for the correlation of rates of transfer and other phenomena," A.I.Ch.E. JI, vol. 18, pp. 1121-1128, 1972.

[25] S. Barcohen and W. Rohsenow, "Thermal optimum spacing of vertical natural convection cooled parallel plates," J. Heat Treansf, vol. 106, pp. 116-123, 1984.

[26] P. M. Teertstra, M. M. Yovanovich, J. R. Culham, and T. F. Lemczyk, "Analytical forced convection modeling of plate fin heat sinks," in Proc. 15th Annu. IEEE Semicon. Thermal Meas. Manag. Symp., San Diego, CA, March 9-11, 1999, pp. 34-41.

[27] W. M. Kays, and A. L. London, Compact Heat Exchangers. New York: McGraw-Hill, 1984.

[28] F. M. White, Fluid Mechanics. New York: McGraw-Hill, 1987.

[29] Y. Muzychka and M. Yovanovich, "Pressure drop in laminar developing flow in noncircular ducts: A scaling and modeling approach," ASME J. Fluids Eng., vol. 131, no. 11, p. 111105, 2009. 